ESAIM: PROCEEDINGS, December 2003, Vol. 13, 1-17

J.P. Penot, Editor

DOI: $10.1051 /$ proc:2003004

\title{
A SURVEY ON ERROR BOUNDS FOR LOWER SEMICONTINUOUS FUNCTIONS
}

\author{
D. AzÉ 1
}

\begin{abstract}
We survey ancient and recent results on global error bounds for the distance to a sublevel set of a lower semicontinuous function defined on a complete metric space. We emphasize the case of a recent characterization of this property which appeared in $[5,7]$. We also review the convex case and show how the known result on sufficient condition for a global error bound can be derived from the quoted characterization.
\end{abstract}

Résumé. Nous revenons sur d'anciens et nouveaux résultats de borne d'erreur pour la distance à un ensemble de sous-niveau d'une fonction semi-continue inférieurement définie sur un espace métrique complet. Nous soulignons en particulier la caractérisation récente de cette propriété apparue dans $[5,7]$. On revient aussi sur le cas convexe, et on montre comment les résultats connus de conditions suffisantes pour une borne globale d'erreur découlent de la caractérisation citée.

\section{INTRODUCTION}

The problem of finding conditions ensuring existence of a global error bound for the distance to a sublevel set of a lower semicontinuous function has been intensively discussed since the seminal work [20] by Hoffman. It can be stated as follows : given a lower semicontinuous function $f: X \rightarrow \mathbb{R} \cup\{+\infty\}$ defined on a complete metric space $X$, we say that $f$ has a global error bound at the level $\alpha$ if there exists a positive real number $\tau$ such that

$$
\tau d(x,[f \leq \alpha]) \leq(f(x)-\alpha)^{+} \text {for all } x \in X,
$$

where $[f \leq \alpha]:=\{x \in X: f(x) \leq \alpha\}, d(x,[f \leq \alpha])$ stands for the distance from $x$ to the set $[f \leq \alpha]$, and $t^{+}=\sup (t, 0)$ denotes the positive part of the real number $t$. In other words the distance to the set $d(x,[f \leq \alpha])$ is at most of the same order than the residual $(f(x)-\alpha)^{+}$. The first step along this line was done by Hoffman in [20] in which (1) was proved for a convex polyhedral function of the type $f(x)=\max _{1 \leq j \leq m}\left(a_{j}^{T} x-b_{j}\right)$ where $a_{1}, \cdots, a_{m} \in \mathbb{R}^{n}$ and $b_{1}, \cdots b_{m} \in \mathbb{R}$, assuming that the polyhedron $[f \leq 0]$ is nonempty. For convex functions, non necessarily polyhedral, a first step was done by Robinson in [38,39] who proved that, assuming that the convex function $f: X \rightarrow \mathbb{R} \cup\{+\infty\}$, defined on a normed space, satisfies the conditions inf $\operatorname{in} f \alpha$ (Slater's condition at the level $\alpha$ ) and $[f \leq \alpha]$ bounded, then (1) holds true. Indeed, assuming that $f\left(x_{0}\right)=\alpha-\theta<\alpha$ with $\theta>0$ and given $x \in X$ with $f(x)>\alpha$, then $x_{t}:=x+t\left(x_{0}-x\right) \in[f \leq \alpha]$ for $t=\frac{f(x)-\alpha}{\theta+f(x)-\alpha} \in[0,1]$ and

$$
\left\|x-x_{0}\right\| \leq\left\|x-x_{t}\right\|+\left\|x_{t}-x_{0}\right\| \leq t\left\|x-x_{0}\right\|+r+\left\|x_{0}\right\|,
$$

1 UMR CNRS MIP, Université Paul Sabatier, 118 Route de Narbonne, 31062 Toulouse cedex, France. e-mail: aze@mip.ups-tlse.fr

(c) EDP Sciences, SMAI 2003 
where $[f \leq \alpha]$ is contained in the closed ball of radius $r$ centered at 0 . Thus we get

$$
d(x,[f \leq \alpha]) \leq\left\|x-x_{t}\right\| \leq t\left\|x-x_{0}\right\| \leq \frac{t}{1-t}\left(r+\left\|x_{0}\right\|\right) \leq \frac{r+\left\|x_{0}\right\|}{\theta}(f(x)-\alpha) .
$$

Later Mangasarian in [28], Auslender-Crouzeix in [1] and Klatte-Li in [25] gave sufficient condition for an error bound in terms of an asymptotic condition which will be discussed later on. Some other sufficient conditions were also given by Deng in $[14,15]$. In the nonconvex case, the first result on error bounds was proved by Ioffe in [21]. Recently Ioffe in [22], Ng-Zheng in [33] and Wu-Ye in [44-46] furnished some sufficient conditions for a global error bound and Penot gave some results in the quasiconvex case in [37]. In the convex case, the first characterization of the existence of a global error bounds seems to be the one given by Cornejo-JouraniZalinescu in [10]. Some other characterization in the convex case were given by Lewis-Pang in [27], by Lemaire in [26] (anticipated by Auslender-Crouzeix-Cominetti in [3]) and by Zalinescu in [47] and recently in [5,45]. In the nonconvex case, one can find a characterization of a global error bound for lower semicontinuous functions defined in a complete metric space in [5, Theorem 2.4] further developed in [7].

Throughout the paper $X$ will be a metric space endowed with the metric $d$, and $f: X \rightarrow \mathbb{R} \cup\{+\infty\}$ be a lower semicontinuous function. For $U \subset X$ and $r \in] 0,+\infty]$ (resp., $r \in\left[0,+\infty\left[\right.\right.$ ), we denote by $B_{r}(U)$ (resp., $\bar{B}_{r}(U)$ ) the open (resp., closed) $r$-enlargement of $U: B_{r}(U):=\{x \in X: d(x, U)<r\}, \bar{B}_{r}(U):=\{x \in X: d(x, U) \leq r\}$, where $d(x, U):=\inf _{y \in U} d(x, y)$, with the convention that $d(x, \emptyset)=+\infty$. If $U=\{x\}$, we simply write $B_{r}(x)$, $\bar{B}_{r}(x)$. In the case where $X$ is a Banach space, we simply write $\bar{B}_{X}$ for $\bar{B}_{1}(0)$. For $\alpha \in \mathbb{R}$ and $\beta \in \mathbb{R} \cup\{+\infty\}$, we let:

$$
[f \leq \alpha]:=\{x \in X: f(x) \leq \alpha\}, \quad[f<\beta]:=\{x \in X: f(x)<\beta\}
$$

denote respectively the closed and open sublevel sets of $f$, and if $\alpha<\beta$, we further denote by $[\alpha<f<\beta]:=$ $[f<\beta] \backslash[f \leq \alpha]$ the slice between $\alpha$ and $\beta$. If $\beta=+\infty$, we shall also write

$$
[f>\alpha]:=[\alpha<f<+\infty], \quad \operatorname{dom} f:=[f<+\infty],
$$

and say, as usual, that $f$ is proper if $\operatorname{dom} f \neq \emptyset$.

We say that a function $f: X \longmapsto \mathbb{R} \cup\{+\infty\}$ satisfies a global error bound between the levels $\alpha$ and $\beta>\alpha$ whenever $[f<\beta] \neq \emptyset$ and there exists a positive real number $\tau$ such that

$$
\tau d(x,[f \leq \alpha]) \leq(f(x)-\alpha)^{+} \text {for all } x \in[f<\beta],
$$

We also say that $f$ satisfies a global error bound at the level $\alpha$ if $(\mathrm{GE})_{\alpha(+\infty)}$ holds true, that is, there exists $\tau>0$ such that

$$
\tau d(x,[f \leq \alpha]) \leq(f(x)-\alpha)^{+} \text {for all } x \in X .
$$

It is noteworthy that, besides its own interest, the question of global error bounds takes equivalent forms which are interesting and sometimes quite surprising. As an example, it is equivalent to the metric regularity of a multifunction. Namely, let $F \subset X \times Y$ be a closed multifunction between metric spaces $X$ and $Y$ identified to its graph. Let $y \in Y$ and let $f_{y}: X \times Y \longrightarrow \mathbb{R} \cup\{+\infty\}$, defined by

$$
f_{y}(x, z)=d(y, z)+i_{F}(x, z)
$$

where $i_{F}$ is the indicator function of $F$, that is $i_{F}(x, y)=\left\{\begin{array}{ll}0 & \text { if }(x, y) \in F \\ +\infty & \text { otherwise }\end{array}\right.$. Then $(\mathrm{GE})_{0}$ leads to

$$
\tau d\left(x, F^{-1}(y)\right) \leq d(y, F(x)) \text { for all } x \in X .
$$


Indeed, one has $\left[f_{y} \leq 0\right]=F^{-1}(y) \times\{y\}$, so that $(\mathrm{GE})_{0}$ yields

$$
\tau d\left((x, z), F^{-1}(y) \times\{y\}\right) \leq d(y, z) \text { for all }(x, z) \in F,
$$

hence, endowing for instance $X \times Y$ with the box distance, xe get

$$
\tau d\left(x, F^{-1}(y)\right) \leq d(y, F(x)) \text { for all } x \in X .
$$

Conversely (3) applied to $F=$ epi $f$ with $y=\alpha$ yields $(\mathrm{GE})_{\alpha}$. The case of metric regularity near a point $\left(x_{0}, y_{0}\right) \in F$, that is the existence of $\tau>0$ such that $\tau d\left(x, F^{-1}(y)\right) \leq d(y, F(x))$ for all $(x, y)$ near $\left(x_{0}, y_{0}\right)$ can also be treated by the means of error bounds (see e.g. $[7,22,23]$ ). One can also observe that finding a global error bound is equivalent to finding a sharp minimum : given $f: X \longrightarrow \mathbb{R} \cup\{+\infty\}$, there exists $\tau>0$ such that

$$
\tau d(x, \operatorname{argmin} f) \leq f(x)-m_{f} \text { for all } x \in X
$$

where $m_{f}=\inf _{X} f$ and $\operatorname{argmin} f=\left[f \leq m_{f}\right]$. Indeed (4) is $(\mathrm{GE})_{m_{f}}$, and conversely $[f \leq \alpha]=\operatorname{argmin}(f-\alpha)^{+}$.

The paper is organised as follows. In section 2, we list some recent sufficient conditions issued from [22] and [45] for the existence of an error bound and we review the results given in [5-7] about the characterization of the existence of a global error bound, along with some local and parametric results. Finally we treat the convex case in section 3 and we check how the known results on sufficient conditions can be derived from the quoted characterizations. Let us mention that, except in Example 1.18, we restrict ourself to first order error bounds, that is we do not treat the case of estimates of the type $\tau d(x,[f \leq \alpha]) \leq\left((f(x)-\alpha)^{+}\right)^{p}$ with $p>1$.

\section{ERROR BOUNDS FOR LOWER SEMICONTINUOUS FUNCTIONS}

In this section, without other indications, $(X, d)$ will denote a complete metric space.

\subsection{Sufficient conditions}

The main tool in what follows is the following version of Ekeland's variational principle.

Proposition 1.1. Let $X$ be complete, $f: X \rightarrow \mathbb{R} \cup\{+\infty\}$ be a (proper) lower semicontinuous function, and let $\bar{x} \in X, \sigma>0$, and $r>0$ be such that :

$$
f(\bar{x})<\inf _{X} f+\sigma r
$$

Then, there exists $x \in B_{r}(\bar{x})$ such that $f(x) \leq f(\bar{x})$ and

$$
f(x)<f(y)+\sigma d(x, y) \quad \text { for every } y \in X \backslash\{x\} .
$$

Among the recent results on existence of an error bound, we firstly quote the following one due to Ioffe in [22, p. 512]. This result is used in the quoted reference to derive metric regularity results for multifunctions.

Lemma 1.2. Let $(X, d)$ be a complete metric space and let $f: X \longmapsto \mathbb{R} \cup\{+\infty\}$ be a proper lower semicontinuous function. Assume that there exist $\bar{x} \in \operatorname{dom} f, \sigma>0, r \in(0,+\infty]$ with $f(\bar{x})<\sigma r$ such that for all $0<t<\sigma$ and for all $x \in[f>0] \cap B_{r}(\bar{x})$, there exists $u \neq x$ with

$$
f^{+}(u)+t d(x, u) \leq f(x)
$$

Then $[f \leq 0] \neq \emptyset$ and $\sigma d(\bar{x},[f \leq 0]) \leq f^{+}(\bar{x})$

The next result is $[45$, Theorem 3$]$ for which we give a simple proof. 
Theorem 1.3. Let $(X, d)$ be a complete metric space and let $f: X \longmapsto \mathbb{R} \cup\{+\infty\}$ be a proper lower semicontinuous function. Assume that $[f<\beta] \neq \emptyset$ and that

$$
\text { for all } x \in[\alpha<f<\beta] \text { there exists } y \in[f \geq \alpha] \backslash\{x\} \text { such that } f(y)+d(x, y) \leq f(x) \text {. }
$$

Then $[f \leq \alpha] \neq \emptyset$, and

$$
d(x,[f \leq \alpha]) \leq(f(x)-\alpha)^{+} \text {for all } x \in[f<\beta] .
$$

Proof. Assume that (7) does not hold. Thus there exists $\bar{x} \in X$ such that $f(\bar{x})<\beta$ and $(f(\bar{x})-\alpha)^{+}<d(\bar{x},[f \leq \alpha])$ so that $\alpha<f(\bar{x})<+\infty$. Let $r>0$ be such that

$$
(f(\bar{x})-\alpha)^{+}<r<d(\bar{x},[f \leq \alpha])
$$

and let $g=(f-\alpha)^{+}$in such a way that $g(\bar{x})<\inf _{X} g+r$. From Proposition 1.1, we can find $x \in X$ such that $d(x, \bar{x})<r, g(x) \leq g(\bar{x})$ and

$$
g(x)<g(y)+d(x, y) \text { for all } y \in X \backslash\{x\} .
$$

Observe that $f(x)>\alpha$ due to $d(x, \bar{x})<r$, and that $f(x)<\beta$ since $g(x) \leq g(\bar{x})$. Returning to (8), we get $x \in[\alpha<f<\beta]$ and $f(x)<f(y)+d(x, y)$ for all $y \in[f \geq \alpha] \backslash\{x\}$, which contradicts (6).

Remark 1.4. In the case where $X$ is a Banach space and $f: X \longrightarrow \mathbb{R} \cup\{+\infty\}$ is convex, then assumption (6) can be weakened under the form

$$
\text { for all } x \in[\alpha<f<\beta] \text { there exists } y \neq x \text { such that } f(y)+d(x, y) \leq f(x) \text {. }
$$

Indeed if $y \neq x$ given by (9) is such that $f(y)<\alpha$, then we can find $t \in] 0,1[$ such that $f(\hat{y})=\alpha$ with $\hat{y}=x+t(y-x)$ and

$$
\frac{f(x)-f(\hat{y})}{\|\hat{y}-x\|} \geq \frac{f(x)-f(y)}{\|y-x\|} \geq 1,
$$

thus (6) holds. It is noteworthy that (9) implies that $(\mathrm{GE})_{\gamma \beta}$ holds for every $\gamma \in[\alpha, \beta[$. We shall see later a converse to this result.

As a consequence, we get the following strengthening [18, Theorem 2] of Takahashi's Theorem in [41]:

Theorem 1.5. Let $(X, d)$ be a complete metric space, let $\varphi: X \longmapsto \mathbb{R} \cup\{+\infty\}$ be a proper lower semicontinuous function, bounded from below and let $\beta>\inf _{X} \varphi$. Assume that, for all $x \in X$ with $\inf _{X} \varphi<\varphi(x)<\beta$, there exists $y \neq x$ such that $\varphi(y)+d(x, y) \leq \varphi(x)$. Then $\operatorname{argmin} \varphi \neq \emptyset$, and

$$
d(x, \operatorname{argmin} \varphi) \leq \varphi(x)-\inf _{X} \varphi \text { for all } x \in[f<\beta]
$$

Proof. Apply Theorem 1.3 with $\alpha=\inf _{X} \varphi$.

Example 1.6. (see [41]) Theorem 1.3 provides a short proof of Nadler's Theorem (in [32]). Let $X$ be a complete metric space, and let $T \subset X \times X$ be a closed-valued multifunction such that $h(T(x), T(y)) \leq k d(x, y)$ for some $0 \leq k<1$ and for all $x, y \in X$ where $h(T(x), T(y))=\max \left(\sup _{z \in T(x)} d(z, T(y)), \sup _{z \in T(y)} d(z, T(x))\right)$ denotes the Hausdorff distance. Let us denote by $F_{T}$ the set of fixed points of $T$, that is the set of those $x \in X$ such that $x \in T(x)$. Let us set $f(x)=d(x, T(x))$, so that $f$ is continuous and $[f \leq 0]=F_{T}$. Let $\varepsilon>0$ be such that $k(1+\varepsilon)<1$, let $x \in[f>0]$ and let $y \in T(x)$ be such that $d(x, y) \leq d(x, T(x))(1+\varepsilon)$. One has $y \neq x$ and $d(y, T(y)) \leq k d(x, y)$ in such a way that

$$
f(x)-f(y)=d(x, T(x))-d(y, T(y)) \geq\left(\frac{1}{1+\varepsilon}-k\right) d(x, y),
$$


thus Theorem 1.3 applies, yielding $F_{T} \neq \emptyset$ and, by letting $\varepsilon$ go to 0 ,

$$
(1-k) d\left(x, F_{T}\right) \leq d(x, T(x)) \text { for all } x \in X
$$

Remark 1.7. a) In Theorems 1.3 and 1.5, one can use $\sigma d$ as a distance with $\sigma>0$.

b) We saw that Theorem 1.5 is an immediate consequence of Theorem 1.3. In fact these two results are equivalent since Takahashi's Theorem is equivalent to Ekeland variational principle, as shown in [11]. In fact Takahashi's Theorem is equivalent to completeness of $X$ (see [41]) and Ekeland's variational principle is also equivalent to completeness of $X$ as shown in [43].

Example 1.8. Theorem 1.3 applies also to the framework of [19]. Let $T$ be a compact topological space, let $\mathcal{C}(T)$ be the space of continuous functions on $T$ equiped with the norm $\|g\|_{\infty}=\max _{t \in T}|g(t)|$, and let $g: \mathbb{R}^{n} \longrightarrow \mathcal{C}(T)$ be a continuous mapping. Let us define $f: \mathbb{R}^{n} \longrightarrow \mathbb{R}$ by $f(x)=\max _{t \in T} g(x)(t)$ and let us consider the set $S:=[f \leq 0]=\left\{x \in \mathbb{R}^{n}: \max _{t \in T} g(x)(t) \leq 0\right\}$. In the quoted reference, it is proved that a global error bound of the type

$$
\tau d(x, S) \leq\left\|g(x)^{+}\right\|_{\infty} \text { for all } x \in \mathbb{R}^{n}
$$

holds true, assuming that the functions $g(\cdot)(t)$ are convex for all $t \in T$ and that the so-called weak Slater condition is in force: there exists $\tau>0, \beta>0$ such that for all $x \in \mathbb{R}^{n}$ with $0<\left\|g(x)^{+}\right\|_{\infty}<\beta$, there exists $y \in \mathbb{R}^{n}$ such that $g(y)(t)<\left\|g(x)^{+}\right\|_{\infty}$ for all $t \in T$ and $\tau\|x-y\|+f(y) \leq f(x)$. It follows that this weak Slater condition implies that (9) holds, and then $f$ has an error bound at the level 0.

\subsection{Characterization of global error bounds}

Let us recall the notion of strong slope introduced by De Giorgi, Marino, and Tosques in [13].

Definition 1.9. Let $f: X \rightarrow \mathbb{R} \cup\{+\infty\}$ be a lower semicontinuous function, and let $x \in \operatorname{dom} f$. Set:

$$
|\nabla f|(x):= \begin{cases}0 & \text { if } x \text { is a local minimum of } f \\ \limsup _{y \rightarrow x} \frac{f(x)-f(y)}{d(x, y)} & \text { otherwise. }\end{cases}
$$

For $x \notin \operatorname{dom} f$, let $|\nabla f|(x):=+\infty$. The nonnegative extended real number $|\nabla f|(x)$ is called the strong slope of $f$ at $x$.

The next result, taken from [7], provides a characterisation of global error bounds for lower semicontinuous functions defined on a complete metric space. This result was previously obtained in [5, Theorem 2.4] in the particular case $\beta=+\infty$.

Theorem 1.10. Let $X$ be complete, $f: X \rightarrow \mathbb{R} \cup\{+\infty\}$ be a lower semicontinuous function, and $\alpha \in \mathbb{R}$, $\beta \in \mathbb{R} \cup\{+\infty\}$ with $\alpha<\beta$.

a) Assume that $[f<\beta] \neq \emptyset$ and

$$
\tau:=\inf _{[\alpha<f<\beta]}|\nabla f|>0
$$

Then

$$
\tau d(x,[f \leq \gamma]) \leq(f(x)-\gamma)^{+} \text {for all } \alpha \leq \gamma<\beta \text { and for all } x \in[f<\beta]
$$

b) Conversely, assume that (11) is satisfied for some $\tau>0$, then $\inf _{[\alpha<f<\beta]}|\nabla f| \geq \tau$. 
Example 1.11. Let $X, Y$ be Banach spaces and let $A: X \longrightarrow Y$ be a linear continuous mapping such that $A(X)=Y$. For $y \in X$, let us introduce the convex continuous function $f: X \longrightarrow \mathbb{R}$ by $f(x)=\|A(x)-y\|$. One has $[f \leq 0]=A^{-1}(y)$. From Baire's Theorem, we can find $\tau>0$ such that $\tau \bar{B}_{Y} \subset \overline{A\left(\bar{B}_{X}\right)}$. Now given $x \in[f>0]$, we can find a sequence $\left(u_{k}\right)_{k \in \mathbb{N}} \subset \bar{B}_{X}$ such that

$$
v:=-\tau\|A x-y\|^{-1}(A(x)-y)=\lim _{k \rightarrow \infty} A\left(u_{k}\right),
$$

yielding

$$
-\tau=\|\cdot\|^{\prime}(A(x)-y ; v)=\lim _{k \rightarrow \infty}\|\cdot\|^{\prime}\left(A(x)-y ; A\left(u_{k}\right)\right)=\lim _{k \rightarrow \infty} f^{\prime}\left(x ; u_{k}\right),
$$

where $g^{\prime}(z ; u)=\lim _{t \downarrow 0} t^{-1}(g(x+t u)-g(x))$ denotes the usual directional derivative of the convex function $g$ at $x$ in the direction $u$. Let $0<\varepsilon<\tau$. We derive that for $k$ large enough we have $f^{\prime}\left(x ; u_{k}\right) \leq-(\tau-\varepsilon)$, from which we get

$$
\tau-\varepsilon \leq-f^{\prime}\left(x ; u_{k}\right)=\left\|u_{k}\right\| \lim _{t \downarrow 0} \frac{f(x)-f\left(x+t u_{k}\right)}{t\left\|u_{k}\right\|} \leq\left\|u_{k}\right\| \limsup _{y \rightarrow x} \frac{f(x)-f(y)}{x-y}=\left\|u_{k}\right\||\nabla f|(x) \leq|\nabla f|(x),
$$

hence $\inf _{[f>0]}|\nabla f|(x) \geq \tau-\varepsilon$. Thus applying Theorem 1.10, we get $(\tau-\varepsilon) d\left(x, A^{-1}(y)\right) \leq\|A(x)-y\|$ for all $x \in X$ and for all $y \in Y$, and then

$$
\tau d\left(x, A^{-1}(y)\right) \leq\|A(x)-y\| \text { for all } x \in X \text { and for all } y \in Y,
$$

which leads immediately to the conclusion of the Banach open mapping theorem.

Remark 1.12. The sufficient condition of $\mathrm{Wu}$ and Ye in Theorem 1.3 is not necessary in order to obtain a global error bound as shown by the following example. Let $X$ be a complete metric space such that

$$
\left|\nabla d_{z}\right|(x)=1 \text { for every } z \in X \text { and for every } x \neq z
$$

where $d_{z}(x)=d(z, x)$. From [7] this property is equivalent to the fact that, for all $\varepsilon>0$ and for all $x, z \in X$ such that $x \neq z$, there exists $y \in X$ such that

$$
\max (d(x, y), d(z, y)) \leq \frac{d(x, z)}{2}+\varepsilon
$$

which is equivalent for $X$ to be a path metric space space (see e.g [17, Theorem 1.8]). A path metric space is a space in which $d(x, z)$ is the infimum of the lengths of the curves joining $x$ and $z$ for all $x \neq z$. A convex metric space, that is a metric space $(X, d)$ for which, for all $x \neq z$, there exists $y \in X$ with $d(x, y)+d(y, z) \leq d(x, z)$, is a path metric space, but there exist path metric spaces which are not convex. Let $X$ be such a metric space. According to Theorem 1.10, and observing that $\left[d_{z} \leq \gamma\right]=\bar{B}_{\gamma}(z)$, property (12) is equivalent to

$$
d\left(x,\left[d_{z} \leq \gamma\right]\right) \leq d(z, x)-\gamma \text { for all } \gamma \geq 0 \text { and for all } x \in X \backslash \bar{B}_{\gamma}(z)
$$

Now let $x \neq z$ be such that $d(x, z)<d(x, y)+d(y, z)$ for all $y \in X \backslash\{x, z\}$. Then for any $0<\gamma<d(x, z)$, the function $d_{z}$ satisfies $(\mathrm{GE})_{\gamma}$, but condition (6) of Theorem 1.3 does not hold due to the fact that $d(x, z)<$ $d(x, y)+d(y, z)$ for all $y \in X \backslash\{x, z\}$, thus $d_{z}(x)<d(x, y)+d_{z}(y)$ for all $x \neq z$ and for all $y \neq x$ so that assumption (6) cannot be satisfied. 


\subsection{Estimates of the strong slope}

In the previous subsection, we gave a characterization of the existence of a global error bound in terms of the strong slope. So it is necessary to estimate the strong slope in order to apply this result. In fact we give two ways for estimate the strong slope. One uses the notion of subdifferential and the other one relies on directional derivatives. We consider here a Banach space $X$ endowed with a norm $\|\cdot\|$, with topological dual $X^{*}, d_{*}$ denoting the metric associated with the norm of $X^{*}$.

We further consider an "abstract" subdifferential operator $\partial$, which associates to any lower semicontinuous function $f: X \rightarrow \mathbb{R} \cup\{+\infty\}$, and any point $x \in X$, a subset $\partial f(x)$ of the (topological) dual $X^{*}$ of $X$, in such a way that $\partial f(x)=\emptyset$ if $x \notin \operatorname{dom} f$, and the following two properties are satisfied:

(P1) if $f$ is convex, then $\partial f(x)=\left\{x^{*} \in X^{*}: f(y) \geq f(x)+\left\langle x^{*}, y-x\right\rangle\right.$ for all $\left.y \in X\right\}$;

(P2) if $g: X \rightarrow \mathbb{R}$ is convex and Lipschitz continuous, and if $\bar{x} \in \operatorname{dom} f$ is a local minimum point of $f+g$ then, for every $\varepsilon>0$ there exist $x, y \in X, x^{*} \in \partial f(x)$, and $y^{*} \in \partial g(y)$ such that

$$
\|x-\bar{x}\| \leq \varepsilon,\|y-\bar{x}\| \leq \varepsilon, \quad f(x) \leq f(\bar{x})+\varepsilon, \text { and }\left\|x^{*}+y^{*}\right\|_{*} \leq \varepsilon .
$$

One easily prove the following (see [22] and also [7]).

Proposition 1.13. Let $X$ be a Banach space and $\partial$ be a subdifferential operator such that properties (P1) and (P2) are satisfied. Then, for every lower semicontinuous function $f: X \rightarrow \mathbb{R} \cup\{+\infty\}$ and every $x \in X$, we have:

$$
|\nabla f|(x) \geq \liminf _{(y, f(y)) \rightarrow(x, f(x))} d_{*}(0, \partial f(y)) .
$$

From the previous proposition, we deduce that $\inf _{U} d_{*}(0, \partial f(x)) \geq \tau \operatorname{implies}_{\inf }|\nabla f|(x) \geq \tau$ for any open subset $U \subset X$. As an immediate consequence of Theorem 1.10 and Proposition 1.13 we thus have:

Corollary 1.14. Let $X$ be a Banach space and $\partial$ be a subdifferential operator such that (P1) and (P2) hold, $f: X \rightarrow \mathbb{R} \cup\{+\infty\}$ be a lower semicontinuous function, and $\alpha \in \mathbb{R}, \beta \in \mathbb{R} \cup\{+\infty\}$ with $\alpha<\beta$ and $[f<\beta] \neq \emptyset$. Assume that, for some $\tau>0$, one has

$$
\inf _{x \in[\alpha<f<\beta]} d_{*}(0, \partial f(x)) \geq \tau
$$

Then, for all $\alpha \leq \gamma<\beta$

$$
\tau d(x,[f \leq \gamma]) \leq(f(x)-\gamma)^{+} \text {for all } x \in[f<\beta]
$$

The preceding corollary extends [44, Theorem 3.1] in two direction. Assumption (P1) and (P2) are less stringent than the one used in the quoted reference, and we do not assume that $[f \leq \alpha]$ is nonempty, but we obtain it as a conclusion. Observe that the Fréchet and the Mordukhovich subdifferential (see e.g [30,31]) satisfies (P1) and (P2) whenever $X$ is an Asplund space. Let us recall that the subdifferential of Mordhukovich $\partial f(x)$ is the set of those $\xi \in X^{*}$ for which there exists sequences $\left(x_{k}\right)_{k \in \mathbb{N}}$ in $X$ such that $\left(\left(x_{k}, f\left(x_{k}\right)\right)_{k \in \mathbb{N}}\right.$ converges to $(x, f(x)),\left(\varepsilon_{k}\right)_{k \in \mathbb{N}}$ in $\left[0,+\infty\right.$ [ converging to 0 and $\left(\xi_{k}\right)_{k \in \mathbb{N}}$ in $X^{*} w^{*}$-converging to $\xi$ such that $\xi_{k} \in \partial_{\varepsilon_{k}}^{F} f\left(x_{k}\right)$, where

$$
\partial_{\varepsilon}^{F} f(z)=\left\{\xi \in X^{*}: \liminf _{y \rightarrow z}\|z-y\|^{-1}(f(y)-f(z)-\langle\xi, y-z\rangle) \geq-\varepsilon\right\}
$$

for $\varepsilon \geq 0$.

Remark 1.15. It is also possible to give a necessary condition for a global error bound in terms of some suitable subdifferential. For example assume that $\partial=\partial_{0}^{F}$ is the Fréchet subdifferential and let $\xi \in \partial f(x)$. As $\liminf _{y \rightarrow x}\|y-x\|^{-1}(f(y)-f(x)-\langle\xi, y-x\rangle) \geq 0$, it follows that

$$
\limsup _{y \rightarrow x}\|y-x\|^{-1}(f(x)-f(y)) \leq\|\xi\|_{*}
$$


yielding $d_{*}(0, \partial f(x)) \geq|\nabla f|(x)$. Assume now that a lower semicontinuous function $f: X \rightarrow \mathbb{R} \cup\{+\infty\}$ is such that for some $\alpha \in \mathbb{R}, \beta \in \mathbb{R} \cup\{+\infty\}$ with $\alpha<\beta$ and $[f<\beta] \neq \emptyset$, we have, for all $\gamma \in[\alpha, \beta[$,

$$
\tau d(x,[f \leq \gamma]) \leq(f(x)-\gamma)^{+} \text {for all } x \in[f<\beta] .
$$

Then Theorem 1.10 tells us that $\inf _{[f>\alpha]}|\nabla f|(x) \geq \tau$, hence $\inf _{x \in[f>\alpha]} d_{*}(0, \partial f(x)) \geq \tau$. Observe that the same method leads to $d_{*}\left(0, \partial f_{\varepsilon}(x)\right) \geq|\nabla f|(x)-\varepsilon$ where $\partial_{\varepsilon} f(x)=\partial_{\varepsilon}^{F} f(x)$ is the $\varepsilon$-Fréchet subdifferential, so that a necessary condition for (14) is also $\inf _{x \in[f>\alpha]} d_{*}\left(0, \partial_{\varepsilon} f(x)\right) \geq \tau-\varepsilon$ for all $\varepsilon>0$. It follows that, when $X$ is finite dimensional, then $\inf _{x \in[f>\alpha]} d_{*}(0, \partial f(x)) \geq \tau$, where $\partial$ is the Mordukhovich subdifferential, is a necessary condition for (14).

The strong slope can also be compared, just using the definitions, with various notions of directional derivative. For example, we have:

$$
\|u\||\nabla f|(x) \geq-f^{\prime}(x ; u) \geq-f^{-}(x ; u) \text { for all } x, u \in X
$$

where

$$
f^{-}(x ; u):=\liminf _{\substack{t \searrow 0 \\ v \rightarrow u}} \frac{f(x+t v)-f(x)}{t}, \quad f^{\prime}(x ; u):=\liminf _{t \searrow 0} \frac{f(x+t u)-f(x)}{t}
$$

are respectively the (lower) contingent and Dini derivative of $f$ at $x$ in the direction $u$.

As a consequence, we derive the following strengthening of [45, Theorem 4] (see also [33, Theorem 2.5]).

Theorem 1.16. Let $X$ be a Banach space and let $f: X \longmapsto \mathbb{R} \cup\{+\infty\}$ be a lower semicontinuous function, and $\alpha \in \mathbb{R}, \beta \in \mathbb{R} \cup\{+\infty\}$ with $\alpha<\beta$ and $[f<\beta] \neq \emptyset$. Assume that there exists $\tau>0$ such that for all $x \in[\alpha<f<\beta]$ there exists $u \in X$ such that $\|u\|=1$ and $f^{\prime}(x ; u) \leq-\tau$. Then,

$$
\tau d(x,[f \leq \gamma]) \leq(f(x)-\gamma)^{+} \text {for all } \alpha \leq \gamma<\beta \text { and for all } x \in[f<\beta]
$$

Conversely, assuming that $X=\mathbb{R}^{n}$ and that (16) is satisfied, then for all $x \in[\alpha<f<\beta]$ there exists $u \in X$ such that $\|u\|=1$ and $f^{-}(x ; u) \leq-\tau$.

Proof. ¿From (15) and Theorem 1.10, we have only to prove the converse. Given $x \in[\alpha<f<\beta]$, there exists a sequence $\left(x_{k}\right)_{k \in \mathbb{N}}$ in $\mathbb{R}^{n}$ such that

$$
\tau \leq|\nabla f|(x)=\lim _{k \rightarrow \infty} \frac{f(x)-f\left(x_{k}\right)}{\left\|x-x_{k}\right\|} .
$$

We can assume that the sequence $u_{k}=\frac{x_{k}-x}{\left\|x_{k}-x\right\|}$ converges to some $u \in \mathbb{R}^{n}$ with $\|u\|=1$. Thus the conclusion follows from

$$
f^{-}(x ; u) \leq \liminf _{k \rightarrow \infty} \frac{f\left(x+t_{k} u_{k}\right)-f(x)}{t_{k}}=-|\nabla f|(x) \leq-\tau,
$$

where $t_{k}=\left\|x_{k}-x\right\|$.

\subsection{Local results}

In some situations (like metric regularity of multifunctions for example), it is useful to have local results at our disposal. We present two examples of this kind of results both taken from [7]. They are quite different since the fact that $[f \leq \alpha] \neq \emptyset$ is an assumption in the first case and is a conclusion in the second one. 
Theorem 1.17. Let $X$ be complete, $f: X \rightarrow \mathbb{R} \cup\{+\infty\}$ be a lower semicontinuous function, $\alpha \in \mathbb{R}, \beta \in$ $\mathbb{R} \cup\{+\infty\}$ with $\alpha<\beta, \emptyset \neq U \subset[f \leq \alpha]$, and $\rho>0$. Assume that

$$
\tau:=\inf _{B_{2 \rho}(U) \cap[\alpha<f<\beta]}|\nabla f|
$$

then, for every $\alpha \leq \gamma<\beta$, we have:

$$
\tau d(x,[f \leq \gamma]) \leq(f(x)-\gamma)^{+} \text {for all } x \in \bar{B}_{\rho}(U) \cap[f<\beta]
$$

Conversely, if (17) is satisfied for all $\alpha \leq \gamma<\beta$, then

$$
\inf _{B_{\rho}(U) \cap[\alpha<f<\beta]}|\nabla f| \geq \tau .
$$

Example 1.18. Corollary 1.14 and Theorem 1.17 contain also the error bounds for lower semicontinuous functions results given by Ngai and Théra in [34, Corollary 3.1]. Let $X$ be an Asplund space and let $\partial$ be the Fréchet subdifferential (which satisfies assumptions $\left(\mathrm{P}_{1}\right)$ and $\left(\mathrm{P}_{2}\right)$ ) and let $f: X \longrightarrow \mathbb{R} \cup\{+\infty\}$ be a proper lower semicontinuous functions. In the quoted reference, it is assumed that for some $p>0$ and $\sigma>0$, one has

$$
\inf _{x \in[f>0]} \inf _{\xi \in \partial f(x)} p(f(x))^{p-1}\|\xi\|_{*} \geq \sigma>0 .
$$

Let $\hat{f}(x)=\operatorname{sgn}(f(x))|f(x)|^{p}$ in such a way that $[\hat{f} \leq 0]=[f \leq 0]$. For any $x \in[\hat{f}>0]=[f>0]$, one easily checks, using the fact that the function $t \mapsto \operatorname{sgn}(t)|t|^{p}$ is increasing and is a diffeomorphism from ]0, $+\infty[$ into ] $0,+\infty[$, that

$$
\partial \hat{f}(x)=p(f(x))^{p-1} \partial f(x) .
$$

Thus (18) is nothing else that $\inf _{x \in[f>0]} d_{*}(0, \partial f(x) \geq \sigma$. Hence Corollary 1.14 leads to

$$
\sigma d(x,[f \leq 0]) \leq\left|[f(x)]_{+}\right|^{p} \text { for all } x \in X .
$$

For the local result, let $\bar{x}$ belonging to the boundary of $[f \leq 0]$ and let $\varepsilon>0$ be such that

$$
\inf _{x \in[f>0] \cap B_{\varepsilon}(\bar{x})} \inf _{\xi \in \partial f(x)} p(f(x))^{p-1}\|\xi\|_{*} \geq \sigma>0 .
$$

Observe that (19) implies that $\inf _{x \in[f>0] \cap B_{\varepsilon}(\bar{x})}|\nabla \hat{f}|(x) \geq \sigma$ (see Proposition 1.13). Then Theorem 1.17 applied with $U=\{\bar{x}\}$ and $\rho=\varepsilon / 2$ says that

$$
\sigma d(x,[f \leq 0]) \leq\left|[f(x)]_{+}\right|^{p} \text { for all } x \in B(\bar{x}, \varepsilon / 2) .
$$

Theorem 1.17 yields a characterization of a weak sharp minimum in the sense of [9] (see [7]). It also allows to characterize the existence of a local error bound. Namely, we have:

Corollary 1.19. Let $X$ be complete, $f: X \rightarrow \mathbb{R} \cup\{+\infty\}$ be a lower semicontinuous function and let $\alpha$, $\beta \in \mathbb{R} \cup\{+\infty\}$ with $\alpha<\beta$, and let $\bar{x} \in[f \leq \alpha] \cap \overline{[\alpha<f<\beta]}$. Then the two following properties are equivalent

a) There exists $r>0$ and $\tau>0$ such that

$$
\tau d(x,[f \leq \gamma]) \leq(f(x)-\gamma)^{+} \text {for all } x \in B_{r}(\bar{x}) \cap[f<\beta] \text { and for all } \alpha \leq \gamma<\beta,
$$

b)

$$
\left\{\begin{array}{l}
\liminf _{x \rightarrow \bar{x}}|\nabla f|(x)>0 . \\
x \in[\alpha<f<\beta]
\end{array}\right.
$$


Proof. Assume that liminf

$$
\left\{\begin{array}{l}
x \rightarrow \bar{x} \\
x \in[\alpha<f<\beta]
\end{array}\right.
$$

$|\nabla f|(x) \geq \tau>0$ and let $0<\sigma<\tau$. Thus there exists $\rho>0$ such

that $\inf _{x \in B_{2 \rho}(\bar{x}) \cap[\alpha<f<\beta]}|\nabla f|(x)>\sigma$. Applying Theorem 1.17 with $U=\{\bar{x}\}$, we obtain that

$$
\sigma d(x,[f \leq \gamma]) \leq(f(x)-\gamma)^{+} \text {for all } x \in \bar{B}_{\rho}(\bar{x}) \cap[f<\beta]
$$

yielding a). Conversely, assuming a), Theorem 1.17 applied again with $U=\{\bar{x}\}$ tells us that

$$
\left\{\begin{array}{l}
\liminf _{x \rightarrow \bar{x}}|\nabla f|(x) \geq \inf _{x \in B_{r}(\bar{x}) \cap[\alpha<f<\beta]}|\nabla f|(x) \geq \tau>0 . \\
x \in[\alpha<f<\beta]
\end{array}\right.
$$

Next theorem is also of local nature. It is well fitted for applications to metric regularity of multifunctions (see [7]).

Theorem 1.20. Let $X$ be complete, $f: X \rightarrow \mathbb{R} \cup\{+\infty\}$ be a lower semicontinuous function, $U$ be a subset of $X, \alpha \in \mathbb{R}$, and $\rho>0$. Assume that $U \cap[f<\alpha+\tau \rho] \neq \emptyset$ and that

$$
\inf _{B_{\rho}(U) \cap[\alpha<f<\alpha+\tau \rho]}|\nabla f| \geq \tau .
$$

Then, $[f \leq \alpha] \neq \emptyset$, and:

$$
\tau d(x,[f \leq \alpha]) \leq(f(x)-\alpha)^{+} \text {for all } x \in U \cap[f<\alpha+\tau \rho]
$$

As a simple illustration, one has the following.

Example 1.21. Let $X, Y$ be Banach spaces, let $\Omega \subset X$ be open, and let $F: \Omega \longrightarrow Y$ be a continuous mapping. Let us assume that $F$ is strictly differentiable at $x_{0} \in U$ and that $A:=D F\left(x_{0}\right)$ is onto in such a way that, for some $\tau>0$, one has $\tau \bar{B}_{Y} \subset A\left(\bar{B}_{X}\right)$. For $0<\varepsilon<\tau$, let $\rho>0$ be such that $F-A$ is $\varepsilon$-Lipschitzian on $\bar{B}_{2 \rho}\left(x_{0}\right) \subset \Omega$. For $y \in X$, let us set $f_{y}(x)=\left\{\begin{array}{ll}\|F(x)-y\| & \text { if } x \in \bar{B}_{2 \rho}\left(x_{0}\right) \\ +\infty & \text { otherwise }\end{array}\right.$, so that $f_{y}$ is lower semicontinuous. Given $x \in B_{2 \rho}\left(x_{0}\right)$, we have $f_{y}^{\prime}(x ; u) \leq\|\cdot\|^{\prime}(F(x)-y ; A(u))+\varepsilon\|u\|$ for all $u \in X$. Assuming $\|F(x)-y\| \neq 0$, we can find $u \in \bar{B}_{X}$ such that $A(u)=-\tau\|F(x)-y\|^{-1}(F(x)-y)$, thus $f_{y}^{\prime}(x ; u) \leq-(\tau-\varepsilon)$. Now let $V$ and $W$ be open neighborhoods of $x_{0}$ and $y_{0}$ such that $f_{y}(x)<(\tau-\varepsilon) \rho$ for all $(x, y) \in V \times W$. Setting $U=B_{\rho}\left(x_{0}\right) \cap V$, it follows that, for all $y \in W$, we have $U \cap\left[f_{y}<(\tau-\varepsilon) \rho\right] \neq \emptyset$ and, using $(15), \inf _{U_{\rho} \cap\left[0<f_{y}<(\tau-\varepsilon) \rho\right]}\left|\nabla f_{y}\right| \geq \tau-\varepsilon$. Thus we can apply Theorem 1.20 with $\alpha=0$ yielding $(\tau-\varepsilon) d\left(x,\left[f_{y} \leq 0\right]\right) \leq f_{y}(x)$ for all $x \in U$ and $y \in W$. As $\left[f_{y} \leq 0\right]=F^{-1}(y) \cap \bar{B}_{2 \rho}\left(x_{0}\right)$, we obtain $(\tau-\varepsilon) d\left(x, F^{-1}(y)\right) \leq\|F(x)-y\|$ for all $x \in U$ and $y \in W$, that is the conclusion of the classical Lyusternik-Graves Theorem.

One can also mention the following parametric result taken from [6, Theorem 3.4].

Theorem 1.22. Let $(X, d)$ be complete, let $P$ be a topological space, let $\Omega \subset P \times X$ open and let $f: P \times X \longrightarrow$ $\mathbb{R} \cup\{+\infty\}$ be a function. Let $\left(p_{0}, x_{0}\right) \in \Omega$ be such that $f\left(p_{0}, x_{0}\right) \leq \alpha$. Assume that $f_{p}:=f(p, \cdot)$ is lower semicontinuous for all $p \in P$, and that $f\left(\cdot, x_{0}\right)$ is upper semicontinuous at $p_{0}$. Let us also assume that, for some $\tau>0$,

$$
\inf _{(p, x) \in[f>\alpha] \cap \Omega}\left|\nabla f_{p}\right|(x) \geq \tau .
$$

Then there exists open subsets $U \ni x_{0}$ and $N \ni p_{0}$ such that $\left[f_{p} \leq \alpha\right] \neq \emptyset$ for all $p \in N$ and

$$
\tau d\left(x,\left[f_{p} \leq \gamma\right]\right) \leq\left(f_{p}(x)-\gamma\right)^{+} \text {for all } \alpha \leq \gamma \text { and }(p, x) \in N \times U
$$


Remark 1.23. In fact the conclusion of Theorem 1.22 is valid under the weaker assumption that $f$ is epi-upper semicontinuous at $\left(p_{0}, x_{0}\right)$, namely: e- $\lim \sup _{p \rightarrow p_{0}} f_{p}\left(x_{0}\right) \leq f_{p_{0}}\left(x_{0}\right)$, where

$$
\text { e- } \limsup _{p \rightarrow p_{0}} f_{p}\left(x_{0}\right)=\sup _{\varepsilon>0} \inf _{N \in \mathcal{N}\left(p_{0}\right)} \sup _{p \in N} \inf _{x \in B_{\varepsilon}\left(x_{0}\right)} f_{p}(x)
$$

\section{The CONVEx CASE}

In this section, $X$ is a Banach space, endowed with a norm $\|\cdot\|$. We denote by $X^{*}$ the topological dual of $X$, and by $d_{*}$ the metric associated with the dual norm. Recall that if $f: X \rightarrow \mathbb{R} \cup\{+\infty\}$ is a convex lower semicontinuous function, the (Fenchel) subdifferential of $f$ at $x \in \operatorname{dom} f$ is given by:

$$
\partial f(x)=\left\{x^{*} \in X^{*}: f(y)-f(x) \geq\left\langle x^{*}, y-x\right\rangle \text { for all } y \in X\right\},
$$

while the directional derivative of $f$ at $x$ in the direction $y \in X$ is:

$$
f^{\prime}(x ; y):=\lim _{t \downarrow 0} \frac{f(x+t y)-f(x)}{t}=\inf _{t>0} \frac{f(x+t y)-f(x)}{t} .
$$

For convex functions, property $(\mathrm{GE})_{\alpha}$ has an interesting consequence.

Proposition 2.1. Let $f: X \longrightarrow \mathbb{R} \cup\{+\infty\}$ be a proper lower semicontinuous function defined on a normed space $X$. Assume that $(G E)_{\alpha}$ holds. Then

$$
N_{[f \leq \alpha]}(x)=\mathbb{R}_{+} \partial f(x)+N_{\text {dom } f}(x) \text { for all } x \in[f=\alpha],
$$

where $N_{[f \leq \alpha]}(x)$ is the usual normal cone to a convex set. In particular,

$$
N_{[f \leq \alpha]}(x)=\mathbb{R}_{+} \partial f(x)
$$

whenever $x \in \operatorname{int}(\operatorname{dom} f)$.

Proof. One obviously has

$$
\mathbb{R}_{+} \partial f(x)+N_{\operatorname{dom} f}(x) \subset N_{[f \leq \alpha]}(x) .
$$

Now, let $\xi \in N_{[f \leq \alpha]}(x)$, so that $x$ minimizes $\langle-\xi, \cdot\rangle$ on $C=[f \leq \alpha]$ and then minimizes $\langle-\xi, \cdot\rangle+c d(\cdot, C)$ for some $c>0$. Thus $x$ minimizes $\langle-\xi, \cdot\rangle+c(f(\cdot)-\alpha)^{+}$on $X$. Then, using a formula on the subdifferential of a finite supremum (see e.g [42])

$$
\xi \in c \partial(f-\alpha)^{+}(x) \subset \mathbb{R}_{+} \partial f(x)+N_{\operatorname{dom} f}(x) .
$$

For convex functions, it is easy to estimate the strong slope (see [7]). One has

$$
|\nabla f|(x)=\sup _{z \in[f<f(x)]} \frac{f(x)-f(z)}{\|x-z\|}=\sup _{z \in[f<f(x)]} \frac{-f^{\prime}(x ; z-x)}{\|x-z\|}=d_{*}(0, \partial f(x)),
$$

at each $x \notin \operatorname{argmin} f$.

Remark 2.2. We observed in Remark 1.4 that a sufficient condition in order to get (GE) $)_{\alpha \beta}$ with $\tau>0$ is that for all $x \in[\alpha<f<\beta]$ there exists $y \neq x$ such that $f(y)+\tau\|y-x\| \leq f(x)$. From Theorem 1.10, a necessary condition for $(\mathrm{GE})_{\alpha \beta}$ is that $\inf _{[\alpha<f<\beta]}|\nabla f|(x) \geq \tau$. Relying on (20) this necessary condition becomes

for all $\hat{\tau}<\tau$ and $x \in[\alpha<f<\beta]$ there exists $y \neq x$ such that $f(y)+\hat{\tau}\|y-x\| \leq f(x)$. 
We now observe in the following that for a convex function, the slope increases with altitude (see [7]). This is proved in [4] in finite dimension under an assumption on the relative interior of the domain of $f$ which is removed here. For $\alpha \in \mathbb{R}$, we let:

$$
[f=\alpha]:=\{x \in X: f(x)=\alpha\} .
$$

Proposition 2.3. Let $X$ be a Banach space, $f: X \rightarrow \mathbb{R} \cup\{+\infty\}$ be a convex, lower semicontinuous function, and $\alpha \in \mathbb{R}$ such that $[f \leq \alpha] \neq \emptyset$. Then:

$$
\inf _{[f>\alpha]}|\nabla f| \geq \inf _{[f=\alpha]}|\nabla f|
$$

Proof. We may assume that $\inf _{[f=\alpha]}|\nabla f|>0$, so that $\alpha>\inf _{X} f$, and that $\inf _{[f>\alpha]}|\nabla f|<+\infty$. Let thus $x \in[f>\alpha]$ and $\sigma>0$ with $\sigma>|\nabla f|(x)$. For $y \in[f \leq \alpha]$, set:

$$
g(y):=f(y)+\sigma\|y-x\|
$$

so that $g>f(x)$ - for, otherwise, let $y \in[f \leq \alpha]$ be such that $f(y)+\sigma\|y-x\| \leq f(x)$, then

$$
|\nabla f|(x) \geq \frac{f(x)-f(y)}{\|x-y\|} \geq \sigma,
$$

a contradiction. Let now $\varepsilon \in] 0, \sigma-|\nabla f|(x)\left[\right.$. According to Proposition 1.1, there exists $y_{\varepsilon} \in[f \leq \alpha]$ such that $g(y) \geq g\left(y_{\varepsilon}\right)-\varepsilon\left\|y-y_{\varepsilon}\right\|$ for all $y \in[f \leq \alpha]$, that is:

$$
f(y)+\sigma\|y-x\| \geq f\left(y_{\varepsilon}\right)+\sigma\left\|y_{\varepsilon}-x\right\|-\varepsilon\left\|y-y_{\varepsilon}\right\| \text { for all } y \in[f \leq \alpha] .
$$

We claim that $f\left(y_{\varepsilon}\right)=\alpha$. For, if $f\left(y_{\varepsilon}\right)<\alpha$, let $\left.z_{\varepsilon} \in\right] x, y_{\varepsilon}\left[\cap[f=\alpha]\right.$; then, writing (22) for $y:=z_{\varepsilon}$ yields:

$$
f\left(z_{\varepsilon}\right)-f\left(y_{\varepsilon}\right) \geq(\sigma-\varepsilon)\left\|z_{\varepsilon}-y_{\varepsilon}\right\|
$$

whence

$$
|\nabla f|(x) \geq \frac{f(x)-f\left(z_{\varepsilon}\right)}{\left\|x-z_{\varepsilon}\right\|} \geq \frac{f\left(z_{\varepsilon}\right)-f\left(y_{\varepsilon}\right)}{\left\|z_{\varepsilon}-y_{\varepsilon}\right\|} \geq \sigma-\varepsilon,
$$

a contradiction. Now, we derive from (22) again that for all $y \in[f \leq \alpha]$ :

$$
f\left(y_{\varepsilon}\right)-f(y) \leq \sigma\left(\|y-x\|-\left\|y_{\varepsilon}-x\right\|\right)+\varepsilon\left\|y-y_{\varepsilon}\right\| \leq(\sigma+\varepsilon)\left\|y-y_{\varepsilon}\right\|,
$$

so that

$$
|\nabla f|\left(y_{\varepsilon}\right)=\sup _{f(y)<f\left(y_{\varepsilon}\right)} \frac{f\left(y_{\varepsilon}\right)-f(y)}{\left\|y_{\varepsilon}-y\right\|} \leq \sigma+\varepsilon,
$$

which shows that $\inf _{[f=\alpha]}|\nabla f| \leq \sigma$, since $\varepsilon$ is arbitrarily small, and the conclusion follows.

The following theorem whose origin dates back to [3] provides a characterization of a global error bound for a convex function. It appeared in [45] and was anticipated in [5] (see also [7]).

Theorem 2.4. Let $X$ be a Banach space, $f: X \rightarrow \mathbb{R} \cup\{+\infty\}$ be a proper, convex, and lower semicontinuous function, and $\alpha \in \mathbb{R}, \beta \in \mathbb{R} \cup\{+\infty\}$ with $\alpha<\beta$ and $[f<\beta] \neq \emptyset$. Then:

$$
\inf _{x \in[\alpha<f<\beta]} \frac{f(x)-\alpha}{d(x,[f \leq \alpha])}=\inf _{x \in[\alpha<f<\beta]} d_{*}(0, \partial f(x)) .
$$

Moreover, if $[f<\beta] \neq \emptyset$, then:

$$
\inf _{x \in[f>\alpha]} \frac{f(x)-\alpha}{d(x,[f \leq \alpha])}=\inf _{x \in[\alpha<f<\beta]} d_{*}(0, \partial f(x)) .
$$


Theorem 2.4 says that $[f \leq \alpha] \neq \emptyset$ whenever $\tau:=\inf _{x \in[\alpha<f<\beta]} d_{*}(0, \partial f(x))>0$ and that $\tau$ is the greatest positive number such that (GE) $)_{\gamma \beta}$ holds for all $\alpha \leq \gamma<\beta$. Moreover, in that case, one has $(\mathrm{GE})_{\gamma \beta}$ implies $(\mathrm{GE})_{\gamma}$.

Of course, we can choose in Theorem 2.4 another estimate of the strong slope $|\nabla f|(x)$ than $d_{*}(0, \partial f(x))$, according to (20). For example, we have the following characterization of the existence of a global error bound, which we state in a "reversed" form. This theorem was proved in [45, Theorem 8] in the reflexive case and in [7] for a general Banach space. The first assertion follows immediately from (9) and (21). The second says that, for convex functions, it is enough to have $(\mathrm{GE})_{\alpha \beta}$ in order to get $(\mathrm{GE})_{\alpha}$.

Theorem 2.5. Let $X$ be a Banach space, $f: X \rightarrow \mathbb{R} \cup\{+\infty\}$ be a proper, convex, and lower semicontinuous function, and $\alpha \in \mathbb{R}, \beta \in \mathbb{R} \cup\{+\infty\}$ with $\alpha<\beta$. Then:

$$
\sup _{x \in[\alpha<f<\beta]} \frac{d(x,[f \leq \alpha])}{f(x)-\alpha}=\sup _{x \in[\alpha<f<\beta]} \inf _{z \in[f<f(x)]} \frac{\|x-z\|}{f(x)-f(z)} .
$$

Moreover, if $[f<\beta] \neq \emptyset$, then:

$$
\sup _{x \in[f>\alpha]} \frac{d(x,[f \leq \alpha])}{f(x)-\alpha}=\sup _{x \in[\alpha<f<\beta]} \inf _{z \in[f<f(x)]} \frac{\|x-z\|}{f(x)-f(z)} .
$$

The preceding theorem can be interpreted in terms of the strong Slater condition. Extending to the case of infinite-valued functions defined on a Banach space the definition of Mangasarian in [29], we say that the proper, convex, lower semicontinuous function $f: X \rightarrow \mathbb{R} \cup\{+\infty\}$ satisfies the strong Slater qualification condition at the level $\gamma \in \mathbb{R}$ if

$$
\sup _{x \in[f=\gamma]} \inf _{z \in[f<\gamma]} \frac{\|x-z\|}{\gamma-f(z)}<+\infty .
$$

According to (20), this is equivalent to say that $\inf _{[f=\gamma]}|\nabla f|>0$ (which is true, in particular, if $[f=\gamma]=\emptyset$.) We also say that $f$ satisfies the strong Slater qualification condition on an interval $] \alpha, \beta[(-\infty<\alpha<\beta \leq+\infty)$ if

$$
\sup _{\alpha<\gamma<\beta} \sup _{x \in[f=\gamma]} \inf _{z \in[f<\gamma]} \frac{\|x-z\|}{\gamma-f(z)}<+\infty,
$$

which is equivalent to $\inf _{[\alpha<f<\beta]}|\nabla f|>0$. Theorem 2.5 says that $f$ has a global error bound between the levels $\alpha$ and $\beta$ if and only if $f$ satisfies the strong Slater qualification condition on $] \alpha, \beta[$, and that if $[f<\beta] \neq \emptyset$, the later condition is equivalent to $f$ having a global error bound above the level $\alpha$.

It is also possible to get a sufficient condition for global error bound dealing with the notion of good asymptotic behaviour of a convex function. Following Auslender and Crouzeix [2] (see also [3]; [10] and [37] for extensions to the non convex case), we recall that the convex lower semicontinuous function $f: X \rightarrow \mathbb{R} \cup\{+\infty\}$ has a good asymptotical behaviour ( $G A B$, for short) if for any sequence $\left(x_{n}\right)$ in $X$ we have:

$$
d_{*}\left(0, \partial f\left(x_{n}\right)\right) \rightarrow 0 \Longrightarrow f\left(x_{n}\right) \rightarrow \inf _{X} f .
$$

This notion naturally provides a verifiable sufficient condition for the existence of global error bounds for $f$. It was proved in [3] and in [10, Theorem 5.2] (this readily follows from the first part of Theorem 2.4 with $\beta=+\infty)$ that $f$ has a good asymptotical behaviour if and only if $\left(\mathrm{GE}_{\alpha}\right)$ holds for any $\alpha>\inf _{X} f$ (recall that " $\alpha>\inf _{X} f$ " reads: " $f$ satisfies the Slater qualification condition at level $\alpha$ ", in the terminology of mathematical programming.) Condition GAB has been used in [8] in order to derive a Hoffman-type estimate in semidefinite optimization.

Combining Theorem 1.10, (20) and Proposition 2.3, we obtain the following useful sufficient condition for a global error bound. 
Theorem 2.6. Let $X$ be a Banach space, $f: X \rightarrow \mathbb{R} \cup\{+\infty\}$ be a convex proper, lower semicontinuous function, and $\alpha \in \mathbb{R}$ such that $[f \leq \alpha] \neq \emptyset$. Then:

$$
\inf _{x \in[f>\alpha]} \frac{f(x)-\alpha}{d(x,[f \leq \alpha])} \geq \inf _{x \in[f=\alpha]} d_{*}(0, \partial f(x))
$$

or equivalently

$$
\sup _{x \in[f>\alpha]} \frac{d(x,[f \leq \alpha])}{f(x)-\alpha} \leq \sup _{x \in[f=\alpha]} \inf _{z \in[f<\alpha]} \frac{\|x-z\|}{\alpha-f(z)}<+\infty .
$$

The preceding theorem says that $(\mathrm{GE})_{\alpha}$ holds true whenever $f$ satisfies the strong Slater qualification at the level $\alpha$. This is a generalization to infinite valued functions defined on a Banach space of the main result in [29].

At this stage, let us list some sufficient conditions ensuring the existence of a global error bound in the convex setting.

- The assumptions of Robinson : $[f<\alpha] \neq \emptyset$ and $[f=\alpha] \subset r \bar{B}_{X}$. Then for $f(x)=\alpha$ and $\xi \in \partial f(x)$ and for $x_{0} \in X$ such that $f\left(x_{0}\right)=\alpha-\theta$ with $\theta>0$, we get

$$
\theta=\alpha-f\left(x_{0}\right)=f(x)-f\left(x_{0}\right) \leq\left\langle\xi, x-x_{0}\right\rangle \leq\|\xi\|_{*}\left\|x-x_{0}\right\| \leq\|\xi\|_{*}\left(r+\left\|x_{0}\right\|\right),
$$

hence

$$
\inf _{f(x)=\alpha} d_{*}(0, \partial f(x)) \geq \frac{\theta}{r+\left\|x_{0}\right\|},
$$

and Theorem 2.6 applies. Observe that it is enough to assume that $[f=\alpha]$ is bounded instead of $[f \leq \alpha]$, and that the obtained estimate is exactly (2) which was derived by elementary computations.

- The assumptions of Deng in [14]: There exists $\tau>0$ and $\|h\|=1$ such that

$$
f^{\infty}(h) \leq-\tau
$$

where $f^{\infty}(h)=\sup _{t>0} t^{-1}(f(x+t h)-f(x))$ for any $x \in \operatorname{dom} f$. Then, for all $x \in \operatorname{dom} f$, one has, using $(20)$

$$
|\nabla f|(x) \geq \frac{f(x)-f(x+t h)}{t\|h\|} \geq \frac{f(x)-f(x+t h)}{t} \geq \tau,
$$

and Theorem 1.10 applies. In fact, it is enough to require that $\|h\| \leq 1$ instead of $\|h\|=1$, and no reflexivity assumption is used as in [14]. This extension to the non reflexive setting can be found in [24, Theorem 3.3].

- The assumptions of Deng in [15]: there exists $\delta>0$ and $0<\Delta$ such that

$$
\sup _{x \in[f \leq \alpha]} d(x,[f \leq \alpha-\delta]) \leq \Delta .
$$

Now given $x \in[f=\alpha]$ and $\varepsilon>0$, we can find $z \in[f \leq \alpha-\delta]$ such that $\|x-z\| \leq \Delta+\varepsilon$, hence for all $\xi \in \partial f(x)$, we get

$$
\delta \leq f(x)-f(z) \leq\langle\xi, x-z\rangle \leq\|\xi\|_{*}\|z-x\| \leq\|\xi\|_{*}(\Delta+\varepsilon),
$$

from which, by letting $\varepsilon \rightarrow 0$,

$$
\inf _{f(x)=\alpha} d_{*}(0, \partial f(x)) \geq \frac{\delta}{\Delta}
$$

and Theorem 2.6 applies again. 
- The assumptions of Mangasarian in [28] and Auslender-Crouzeix in [1]: $X=\mathbb{R}^{n},[f<\alpha] \neq \emptyset$ and, for any sequence $\left(x_{k}\right)_{k \in \mathbb{N}}$ in $[f=\alpha]$ such that $\lim _{k \rightarrow \infty}\left\|x_{k}\right\|=+\infty, 0$ is not limit of a sequence $\left(\xi_{k}\right)_{k \in \mathbb{N}}$ with $\xi_{k} \in \partial f\left(x_{k}\right)$. Then, it follows that

$$
\inf _{f(x)=\alpha} d(0, \partial f(x))>0
$$

Indeed, assume on the contrary, that there exist sequences $\left(x_{k}\right)_{k \in \mathbb{N}}$ in $[f=\alpha]$ and $\left(\xi_{k}\right)_{k \in \mathbb{N}}$ with $\xi_{k} \in$ $\partial f\left(x_{k}\right)$ such that $\lim _{k \rightarrow \infty} \xi_{k}=0$. From our assumption $\left(x_{k}\right)_{k \in \mathbb{N}}$ is bounded, and then

$$
0=\lim _{k \rightarrow \infty} \xi_{k}^{T} x_{k} \geq \alpha+\liminf _{k \rightarrow \infty} f^{*}\left(\xi_{k}\right) \geq \alpha+f^{*}(0)
$$

hence $\inf _{X} f \geq \alpha$, a contradiction. Thus we can use Theorem 2.6.

Let us conclude with some bibliographical comments. To our knowledge, the first characterization of the existence of a global error bound in the convex case is [10, Theorem 5.1], where it is shown that if $\operatorname{argmin} f \neq \emptyset$, $\alpha:=\min _{X} f$ and $\sigma>0$, then

if and only if

$$
f(x)-\alpha \geq \sigma d(x,[f \leq \alpha]) \quad \text { for all } x \in X
$$

$$
\partial f^{*}\left(x^{*}\right) \subset \partial f^{*}(0) \text { for all } x^{*} \in X^{*} \text { such that }\left\|x^{*}\right\|_{*}<\sigma,
$$

where $f^{*}$ denotes the Fenchel conjugate of $f$. In fact, as $x \in \partial f^{*}\left(x^{*}\right)$ if and only if $x^{*} \in \partial f(x)$, the latter condition is clearly equivalent to $\inf _{x \in[f>\alpha]} d_{*}(0, \partial f(x)) \geq \sigma$. This result was stated under this form in [26, Proposition 3.1 and 7.1] with $\alpha \in \mathbb{R}$ (more recently, see also [47, Corollary 2.2].) Recall that in Theorem 2.4, the fact that $[f \leq \alpha] \neq \emptyset$ is a consequence of the fact that $\inf _{x \in[f>\alpha]} d_{*}(0, \partial f(x))>0$. Lewis and Pang gave in [27] (extended to the reflexive case in [47]) a characterization in the convex case and finite dimensions, namely there exists $\tau>0$ such that

$$
\inf _{[f=\alpha]} \inf _{u \in N_{[f \leq \alpha]}(x)} f^{\prime}(x ; u) \geq \tau\|u\| .
$$

Observe that (23) implies $\inf _{x \in[\alpha<f]} d_{*}(0, \partial f(x)) \geq \tau$. Indeed, if $\xi \in \partial f(x)$ where $x \in[f>0]$, then, denoting by $\bar{x}$ the projection of $x$ on $[f \leq \alpha]$, we have $x-\bar{x} \in N_{[f \leq \alpha]}(\bar{x})$, and

$$
\tau\|x-\bar{x}\| \leq f^{\prime}(\bar{x}, x-\bar{x}) \leq f(x)-f(\bar{x}) \leq \xi^{T}(x-\bar{x}) \leq\|\xi\|\|x-\bar{x}\|,
$$

whence $\|\xi\| \geq \tau$. The fact that

$$
\inf _{x \in[f>\alpha]} \frac{f(x)-\alpha}{d(x,[f \leq \alpha])}=\inf _{x \in[\alpha<f]} d_{*}(0, \partial f(x))
$$

and that $[f \leq \alpha] \neq \emptyset$ is a consequence of $\inf _{x \in[\alpha<f]} d_{*}(0, \partial f(x))>0$ appeared simultaneously in $[5,45]$. Theorem 2.4 with the slice $[\alpha<f<\beta]$ is due to $\mathrm{Wu}$ and Ye in [45]. Theorem 2.5 is taken from [7] and was previously given in [45] in the reflexive case. Theorem 2.6 (see [7]) is a generalization to the general Banach space setting and to possibly non continuous convex functions of a result of Mangasarian in [29].

\section{REFERENCES}

[1] A. Auslender and J.-P. Crouzeix, Global regularity theorems, Math. Oper. Res. 13 (1988), no. 2, $243-253$.

[2] A. Auslender and J.-P. Crouzeix, Well behaved asymptotical convex functions, Ann. Inst. Henri Poincaré, Anal. Non Linéaire 6 (1989), 101-121.

[3] A. Auslender, R. Cominetti, and J.-P. Crouzeix, Convex functions with unbounded level sets, SIAM J. Optim. 3 (1993), $669-687$.

[4] A. Auslender and M. Teboulle, Asymptotic Cones and Functions in Optimization and Variational Inequalities, Springer Monographs in Mathematics, Springer-Verlag, New York, 2003. 
[5] D. Azé and J.-N. Corvellec, On the sensitivity analysis of Hoffman constants for systems of linear inequalities, SIAM J. Optim. 12 (2002), 913-927.

[6] D. Azé, J.-N. Corvellec, and R. E. Lucchetti, Variational pairs and applications to stability in nonsmooth analysis, Nonlinear Anal. 49 (2002), 643-670.

[7] D. Azé and J.-N. Corvellec, Characterizations of error bounds for lower semicontinuous functions on metric spaces, preprint University of Toulouse, 2003.

[8] D. Azé and J.-B. Hiriart-Urruty, Optimal Hoffman-type estimates in eigenvalue and semidefinite inequality constraints, J. Global Optim. 24 (2002), 133-147.

[9] J. V. Burke and M. C. Ferris, Weak sharp minima in mathematical programming, SIAM J. Control Optim. 31 (1993), 13401359 .

[10] O. Cornejo, A. Jourani, and C. Zălinescu, Conditioning and upper-Lipschitz inverse subdifferentials in nonsmooth optimization problems, J. Optim. Theory Appl. 95 (1997), 127-148.

[11] P. Daffer, H. Kaneko and W. Li, Variational Principle and Fixed Points, in Set Valued Mappings and Applications to Nonlinear Analysis, Edited by R. P. Agarwal and D. O'Regan, Taylor and Francis Publ. (2002).

[12] J.-P. Dedieu, Approximate solutions of analytic inequality systems, SIAM J. Optim. 11 (2000), no. 2, 411-425.

[13] E. De Giorgi, A. Marino, and M. Tosques, Problemi di evoluzione in spazi metrici e curve di massima pendenza (Evolution problems in metric spaces and curves of maximal slope, Atti Accad. Naz. Lincei Rend. Cl. Sci. Fis. Mat. Natur. 68 (1980), $180-187$.

[14] S. Deng, Computable error bounds for convex inequality systems in reflexive Banach spaces, SIAM J. Optim. 7 (1997), no. 1, $274-279$.

[15] S. Deng, Global error bounds for convex inequality systems in Banach spaces, SIAM J. Control Optim. 36 (1998), no. 4, $1240-1249$.

[16] I. Ekeland, On the variational principle, J. Math. Anal. Appl. 47 (1974), 324-353.

[17] M. Gromov, Metric structures for Riemanian and non-Riemanian spaces, Birkhäuser, Progress in Mathematics, Boston, 1999.

[18] A. Hamel, Remarks to an equivalent formulation of Ekeland's variational principle, Optimization 31 (1994), no. 3, $233-238$.

[19] M. Gugat, Error bounds for infinite systems of convex inequalities without Slater's condition, Math. Program. 88 (2000), no. 2, Ser. B, 255-275.

[20] A. J. Hoffman, On approximate solutions of systems of linear inequalities, J. Res. Nat. Bur. Stand. 49 (1952), $263-265$.

[21] A. Ioffe, Regular points of Lipschitz functions, Trans. Amer. Math. Soc. 251 (1979), 61-69.

[22] A. Ioffe, Metric regularity and subdifferential calculus, Russian Math. Surveys 55 (2000), no. 3, 501-558.

[23] A. Ioffe, Towards metric theory of metric regularity, in Approximation, Optimization and Mathematical Economics (Proc. Fifth Int. Conf. on Approximation and Optimization in the Caribbean, Guadeloupe, 1999), M. Lassonde Ed., Physica-Verlag, Heidelberg, 2001, 165-176.

[24] A. Jourani, Hoffman's error bound, local controllability, and sensitivity analysis, SIAM J. Control Optim. 38 (2000), no. 3, 947-970.

[25] D. Klatte, W. Li, Asymptotic constraint qualifications and global error bounds for convex inequalities, Math. Program. 84 (1999), no. 1, Ser. A, 137-160.

[26] B. Lemaire, Well-posedness, conditioning and regularization of minimization, inclusion and fixed-point problems, Pliska Stud. Math. Bulgar. 12 (1998), 71-84.

[27] A. S. Lewis and J. S. Pang, Error bounds for convex inequality systems, Nonconvex Optim. Appl., J.-P. Crouzeix et al. Eds., Kluwer Acad. Publ. 27, 1998, 75-110.

[28] O. L. Mangasarian, A condition number for differentiable convex inequalities, Math. Oper. Res. 10 (1985), no. 2, 175-179

[29] O. L. Mangasarian, Error bounds for nondifferentiable convex inequalities under a strong Slater constraint qualification, Math. Program. 83 (1998), 187-194.

[30] B. S. Mordukhovich, Metric approximations and necessary optimality conditions for general classes of nonsmooth extremal problems, Soviet Math. Dokl. 22 (1980), 526-530.

[31] B. S. Mordukhovich and Y. Shao, Differential characterizations of covering, metric regularity and Lipschitzian properties of multifunctions, Nonlinear Anal. 25 (1995), 1401-1428.

[32] S.B. Nadler, Multivalued contraction mappings, Pac. J. Math. 30 (1969), 475-488.

[33] K. F. Ng and X. Y. Zheng, Error bounds for lower semicontinuous functions in normed spaces, SIAM J. Optim. 12 (2001), $1-17$.

[34] H.V. Ngai and M. Théra, Error bounds for systems of lower semicontinuous functions in Asplund spaces and applications, preprint, 2003.

[35] H.V. Ngai and M. Théra, Error bounds for convex differentiable inequalities systems in Banach spaces, preprint, 2003.

[36] J.S. Pang, Error bounds in mathematical programming, Math. Programming 79 (1997), no. 1-3, Ser. B, $299-332$.

[37] J.-P. Penot, Well-behavior, well-posedness and nonsmooth analysis. Proceedings of the 4th International Conference on Mathematical Methods in Operations Research and 6th Workshop on Well-posedness and Stability of Optimization Problems (Sozopol, 1997), Pliska Stud. Math. Bulgar. 12 (1998), 141-190.

[38] S.M. Robinson, An application of error bounds for convex programming in a linear space, SIAM J. Control 13 (1975), $271-273$. 
[39] S.M. Robinson, Stability theory for systems of inequalities. II. Differentiable nonlinear systems, SIAM J. Numer. Anal. 13 (1976), no. 4, 497-513.

[40] M. Studniarski and D. E. Ward, Weak sharp minima: characterizations and sufficient conditions, SIAM J. Control Optim. 38 (1999), 219-236.

[41] W. Takahashi, Existence theorems generalizing fixed point theorems for multivalued mappings, Fixed point theory and applications (Marseille, 1989), 397-406, Pitman Res. Notes Math. Ser., 252, Longman Sci. Tech., Harlow, 1991.

42] M. Volle, On the subdifferential of an upper envelope of convex functions, Acta Math. Vietnam. 19 (1994), no. 2, $137-148$.

[43] J.D. Weston, A characterization of metric completeness, Proc. Amer. Math. Soc. 64 (1977), 186-188.

[44] Z. Wu and J. Ye, Sufficient conditions for error bounds, SIAM J. Optim. 12 (2001/02), no. 2, 421-435.

[45] Z. Wu and J. Ye, On error bounds for lower semicontinuous functions, Math. Program. 92 (2002), 301-314.

[46] Z. Wu and J. Ye, First-order and second-order conditions for error bounds, preprint 2002.

[47] C. Zălinescu, Weak sharp minima, well behaving functions and global error bounds for convex inequalities in Banach spaces, Proc. 12-th Baikal Int. Conf. on Optimization Methods and their Applications, V. Bulatov and V. Baturin Eds., Institute of System Dynamics and Control Theory of SB RAS, Irkutsk, 2001, 272-284.

[48] C. Zălinescu, Convex Analysis in General Vector Spaces, World Scientific Publ. Co., River Edge, NJ, 2002. 